\title{
Colored Shade Nets can Relieve Abnormal Fruit Softening and Premature Leaf Senescence of "Jumeigui" Grapes during Ripening Under Greenhouse Conditions
}

Qian Zha ( $\square$ zhaqian1988@163.com )

Shanghai Academy of Agricultural Sciences

\section{Xiaojun Xi}

Shanghai Academy of Agricultural Sciences

Xiangjing Yin

Shanghai Academy of Agricultural Sciences

Yani He

Shanghai Academy of Agricultural Sciences

Aili Jiang

Shanghai Academy of Agricultural Sciences

\section{Research Article}

Keywords: Abnormal softening, colored shade net, diurnal temperature, grape, premature leaf senescence

Posted Date: September 21st, 2021

DOI: https://doi.org/10.21203/rs.3.rs-902508/v1

License: (c) (1) This work is licensed under a Creative Commons Attribution 4.0 International License.

Read Full License 


\section{Abstract}

When grapes reach maturity, they usually experience extremely high-temperature periods in southern China, causing premature leaf senescence, abnormal fruit softening, and fruiting period shortening. Their quality and production efficiency are also severely affected. 'Jumeigui' grapes were examined in terms of fruit quality and leaf senescence under shading treatments; green, blue, black, and gray aluminum foil nets were used for shading, and their spectra were measured. At the same density, shade net color significantly affected cooling and shading efficiencies, and gray net had the best light transmission and cooling effect. Shading treatment significantly alleviated abnormal grape softness during hot periods. Total soluble solids (TSS) content and grape coloration were affected under gray, blue, and green shade nets. TSS exceeded $18 \%$ under gray, blue, and green nets, meeting the requirements of first-class highquality fruit. However, peel coloration was not notably affected under gray and blue shade nets, while the non-shading treatment produced clear heat-stress damage, especially on the edges of old leaves. The net photosynthetic rate of the bottom five old leaves under the non-shading treatment was significantly lower than that under the shading treatment, indicating that high light and heat caused premature leaf senescence. In summary, colored shade nets can reduce the temperature and light in the greenhouse, while alleviating premature senescence of perennial grape plants. However, the quality of the grapes treated using black shade nets was poor; superior quality was achieved using gray and blue shade nets. These results can be applied in future cultivation facilities during high-temperature periods.

\section{Background}

Recently, the area under grape cultivation in China has significantly expanded with the vigorous promotion of cultivation techniques, and southern China has become the main production area for table grapes. The optimum temperature range for grape development is $25-35^{\circ} \mathrm{C}^{1}$. However, the ripening phase of greenhouse grapes in the southern region usually takes place during an extremely hot period $\left(\sim 45^{\circ} \mathrm{C}\right)$ in open fields ${ }^{2}$. The effect of the sun on the composition of grapes can be significant and complex ${ }^{3}$ : solar radiation and heat can affect the metabolic reaction rates indirectly via dehydration, or directly via heat stress.

In China, Vitis vinifera L. 'Jumeigui' variety is widely cultivated for fresh grape production ${ }^{4}$. It has the advantages of good fruit setting, strong fragrance, and moderate sweetness and sourness, but is prone to experiencing extremely hot periods during the mature stage. This climate change causes abnormal fruit softening and premature senescence of the vine leaves, which shortens the fruit-bearing period, severely affecting the quality and production efficiency of the grapes. Many cooling methods and technologies have been proposed to reduce the effect of sunlight during crop production. Shade net covering is the most widely used approach, due to its low cost and simple operation ${ }^{5}$. However, during the cooling process, if the shade net type is not carefully selected and does not allow the plants to maintain effective photosynthesis, the shading will affect plant growth ${ }^{6}$ and reduce productivity during summer. Recently, colored shade nets have been introduced and have become widely applied in the industry. These nets can 
filter the sunlight spectrum and disperse the direct light through a special component that promotes crop growth ${ }^{7}$.

Warren et al. ${ }^{8}$ reported that the growth and yield of horticultural crops in growth facilities can be strongly dependent on light, and it is generally understood that shading affects grape quality ${ }^{9}$. The use of shading nets will reduce the light intensity, so incorrect use of shading nets can have a negative impact ${ }^{6}$. Hence, we aimed to use colored shade nets and thus identify sunshade materials with a higher cooling and lighttransmittance effect, to achieve high-quality grape production. This will be particularly important climate change causes global temperatures to rise.

\section{Materials And Methods}

\section{Plant material and treatments.}

The experiments were performed in July to September 2020, at the Zhuanghang (ZH) experimental vineyard, Shanghai Academy of Agricultural Sciences, Shanghai, China $\left(30^{\circ} 51^{\prime} \mathrm{N}, 121^{\circ} 13^{\prime} \mathrm{E}\right)$ and at Shanghai Pingqi (PQ) Grape Planting Professional Cooperative $\left(30^{\circ} 11^{\prime} \mathrm{N}, 121^{\circ} 25^{\prime} \mathrm{E}\right)$, using the table grape cultivar 'Jumeigui'. The study site has a subtropical monsoon climate. Due to the high rainfall levels in this area, grapes are planted under plastic film coverings to reduce the incidence of plant disease and insect pests. Facility agriculture ( $\mathbb{Q} \mathbb{W}_{\mathbb{Q}}$ or shèshī nóngyè in Chinese) is a production method that uses clustered plastic-roofed greenhouses and irrigation to produce crops ${ }^{10}$. The own-rooted grapevines were planted in the spring of 2008 in a north-south orientation at a $4 \mathrm{~m} \times 2.8 \mathrm{~m}$ spacing. The vines were grown in a rain shelter with a Y-shaped training system at ZH $(8 \mathrm{~m} \cdot 45 \mathrm{~m})$ and a flat scaffolding system at $P Q(6 \mathrm{~m} \cdot 41 \mathrm{~m})$. The vines were managed according to standard viticulture and disease control practices.

Blue, green, and black shade nets and aluminum foil gray shade nets were purchased from an agricultural market (Jiehang Agricultural Materials Factory, Yangzhou, Jiangsu, China). The colored shade nets $(6 \mathrm{~m} \cdot 41 \mathrm{~m}$ at $\mathrm{PQ}, 8 \mathrm{~m} \cdot 45 \mathrm{~m}$ at $\mathrm{ZH})$ were used to cover the single-shed facilities. The experiment adopted a random block design and was repeated three times. For the control, no shade net was used. The processing period was from July 24, 2020 (50\% color change) to September 3, 2020. Fruit harvesting occurred on August 18,2020, and leaf-related indicators were measured on September 3 , 2020. The grapes were judged to be mature when the total soluble solid content (TSS) was higher than 16 Brix. The grape harvest period occurred when the grapes were mature, and the leaf photosynthetic performance measurement period occurred after the grape harvest. RC-4HC (Jiangsu Jingchuang Electric Co., Ltd., Nanjing, China), which placed near the berries, was used to record the temperature. All methods were performed in accordance with the relevant guidelines and regulations.

\section{Spectral analysis.}


A PMS-2000 UV-VIS-near IR spectrophotocolorimeter (Everfine Co., Ltd., Hangzhou, China) was used to analyze the physical properties of the transmitted light. Spectral data analysis of transmitted light was performed under different color shading nets, and the analysis wavelength range used was $300-800 \mathrm{~nm}$.

\section{Total soluble solids (TSS) measurement.}

For each treatment, 30 grape berries from the top, middle, and bottom of ten bunches were prepared, and the TSS were determined after pressing the juice separately. TSS was measured in degrees Brix using a PAL-1 digital refractometer (Atago, Tokyo, Japan).

\section{Fast chlorophyll a fluorescence kinetic parameters.}

A Pocket-PEA fluorimeter (Plant Efficiency Analyzer, Hansatech Instruments Ltd., King's Lynn Norfolk, UK) was used to determine the fast chlorophyll a fluorescence kinetics of the grape leave ${ }^{11}$. The definitions of parameters are shown in Table S1.

\section{Leaf gas-exchange parameters.}

Photosynthetic measurements were performed by selecting five old lower leaves near the base and five adult upper leaves near the top. The gas-exchange parameters comprised the net photosynthetic rate $(A)$, stomatal conductance $(g s)$, transpiration rate $(E)$, and intercellular $\mathrm{CO}_{2}$ concentration $(C I)$ of the leaves, which were measured using a portable photosynthetic CIRAS-3 system (PP Systems, Amesbury, MA, USA $)^{12}$.

\section{Sugar analysis.}

For this analysis, ten berries were selected from the top, middle, and bottom of three bunches and mixed. The pulps were ground with liquid nitrogen prior to further use. Each treatment was replicated three times. The extraction of soluble sugars was performed and determined via high-performance liquid chromatography, using the Waters E2695 system (Waters, Milford, MA, USA), as described by Zha et al. ${ }^{11}$.

\section{Anthocyanin content analysis.}

For this analysis, ten berries were selected from the top, middle, and bottom of three bunches and mixed. The skins were ground with liquid nitrogen prior to further use. Each treatment was replicated three times. Anthocyanin content was determined using the $\mathrm{pH}$-differential method ${ }^{13,14}$. 


\section{Classifying symptoms of abnormal softening.}

We examined the abnormal softening of the grapes in the field, and developed a grading standard where we investigated 30 clusters per treatment and estimated the softening percentage using specific equations. Softening was graded according to the following scale: level 0 , no abnormal softening symptoms (N1); level 1, abnormal softening up to $10 \%$ (N2); level 2, abnormal softening up to $30 \%$ (N3); level 3, abnormal softening up to $50 \%$ (N4); level 4, abnormal softening up to $70 \%$ (N5); and level 5 , abnormal softening up to $100 \%$ (N6).

Softening index $=(0 \cdot N 1+1 \cdot N 2+2 \cdot N 3+3 \cdot N 4+4 \cdot N 5+5 \cdot N 6) /[5 \cdot(N 1+N 2+N 3+N 4+N 5+N 6)]$ 0 to 5 refers to the plant replicate number.

\section{Grape texture analysis.}

For each treatment, 30 grape berries from the top, middle, and bottom of ten bunches were prepared, and the berry texture was analyzed using a TA.XT.Plus type physical property tester (Stable Micro System, Godalming, UK). Fruit firmness was expressed as the force $(\mathrm{N})$ required to deform the berries, according to Lijavetzky et al. ${ }^{15}$ (2012).

\section{Berry skin color.}

For each treatment, 30 grape berries from the top, middle, and bottom of ten bunches were prepared, and berry color was analyzed using a hand-held C410 Chroma Meter (Konica Minolta, Chiyoda-ku, Tokyo, Japan) at the equatorial portion of each berry. The Color Index of Red Grapes (CIRG), which is based on CIELab data, was calculated using the formula CIRG $=(180-h) /\left(L^{*}+C^{*}\right)^{11}$. The index used for the evaluation of the appearance and color of the berry was: $\mathrm{CIRG}<2$ represents yellow-green, $2<\mathrm{CIRG}<4$ pink, $4<$ CIRG $<5$ red, $5<$ CIRG $<6$ deep red, and CIRG $>6$ blue-black $^{16}$ (Amiri et al., 2010).

\section{Statistical analysis.}

The TSS and CIRG data was tested to confirm that they were normally distributed. These data were compared through two dimensions, average and distribution. The frequency distributions were generated using the function "NORMDIST" in Microsoft Excel 2010 (Redmond, WA, USA). The differences among treatments were assessed using a one-way ANOVA followed by the Tukey-test in SPSS v22.0 (IBM Corp., Armonk, NY, USA).

\section{Results}




\section{Temperature and light transmittance.}

Non-shading treatment often has temperatures of $40^{\circ} \mathrm{C}$ from July to September. The temperature in the greenhouse was significantly reduced after using the colored shade nets (Fig. 1A). Table 1 summarizes the temperature differences between the non-shading and shading treatment. The black shade net produced the largest temperature drop $\left(0.8-7.6^{\circ} \mathrm{C}\right)$, followed by the gray, blue, and green nets, with temperature reductions of $1.2-7^{\circ} \mathrm{C}, 0.7-6.1^{\circ} \mathrm{C}$, and $0.4-6.1^{\circ} \mathrm{C}$, respectively. This indicates that the cooling effect of the black and gray shade nets was more effective than that of the blue and green nets. The determination of absolute spectral value of the colored shade nets in the wavelength range of 300-800 $\mathrm{nm}$ is shown in Fig. 1B; the strongest absorption regions of chlorophyll were 430-450 nm and 640-660 $\mathrm{nm}$, where the absolute spectral value of colored shade net was significantly reduced. Light transmittance $(300-800 \mathrm{~nm})$ was higher under the blue $(57.3 \%)$ and gray $(46.2 \%)$ shade nets than the Green $(40 \%)$ and Black (17.1\%) shade nets (Table 2). The leaves were prone to withering and premature aging without the shading treatment, which affected the appearance of the grapes (Figure S1). Peel color was best under the non-shading treatment, followed by blue and gray, with the poorest color under the black shade net (Fig. 2A and 2E).

Table 1

Temperature difference between the non-shading and shading treatments (using gray, green, blue, or black net).

\begin{tabular}{|lllll|}
\hline Temperature $\left({ }^{\circ} \mathrm{C}\right)$ & GRAY & GREEN & BLUE & BLACK \\
\hline$\Delta \min$ & 1.2 & 0.4 & 0.7 & 0.8 \\
\hline$\Delta \max$ & 7 & 6.1 & 6.1 & 7.6 \\
\hline
\end{tabular}

Table 2

Light transmittance of the colored shade net (gray, green, blue, or black) at different wavelengths. This was calculated by dividing the sum of the values of the shading treatment transmission spectra in the $430-450 \mathrm{~nm}$ range by the sum of the values of the solar spectrum in the same range.

\begin{tabular}{|lllll|}
\hline Wavelength Range $(\mathrm{nm})$ & GRAY & GREEN & BLUE & BLACK \\
\hline $300-800$ & 46.2 & 40.00 & 57.3 & 17.1 \\
\hline $430-450$ & 46.1 & 30.82 & 63.0 & 14.1 \\
\hline $640-660$ & 46.4 & 28.84 & 48.9 & 16.6 \\
\hline
\end{tabular}

\section{Berries internal and external qualities.}


We used conventional methods to determine and analyze fructose, glucose, and anthocyanin content (Fig. 2); they were reduced to varying degrees at the two study sites, indicating that the shading treatment affects grape appearance and sugar content. However, these data only included three non-biological replicates; each replicate was a mixture of ten samples, and the average value was determined. Furthermore, 30 grape berries were selected to determine the TSS, CIRC, and others; the data were objectively represented as frequency distribution.

Based on the method used to evaluate disease and heat damage, we designed a method to subjectively evaluate fruit softening, expressed as an index (Table 3); the higher the softening index, the softer the fruit. Further, the "Jumeigui" fruit softened abnormally without shading, with a fruit softening index of $72-73.33 \%$. Hence, using colored shade nets can significantly alleviate abnormal fruit softening. The softening index decreased to $23.33-31.33 \%$ and $35.33-59.33 \%$ at $P Q$ and $Z \mathrm{H}$, respectively. Subsequently, the texture analyzer was used to objectively evaluate fruit firmness.

Table 3

Softening index of the 'Jumeigui' grape cultivar.

\begin{tabular}{|lll|}
\hline \multirow{2}{*}{ Treatment } & Softening index (\%) \\
\cline { 2 - 3 } & PQ & ZH \\
\hline NONE & 73.33 & 72 \\
\hline GRAY & 26.67 & 43.33 \\
\hline BLUE & 23.33 & 42.67 \\
\hline GREEN & 31.33 & 59.33 \\
\hline BLACK & 26 & 35.33 \\
\hline $\begin{array}{l}\text { NONE: no shading; GRAY, BLUE, GREEN, and BLACK: shade net colors. PQ and ZH: experimental } \\
\text { vineyard sites at the Shanghai Pingqi Grape Planting Professional Cooperative (30 } \\
\text { and at Zhuanghang, the site at the Shanghai Academy of Agricultural Sciences, Shanghai, China } \\
\left.\text { (30 } 25^{\circ} 51^{\prime} \mathrm{N}, 121^{\circ} 13^{\prime} \mathrm{E}\right) \text {, respectively. }\end{array}$ \\
\hline
\end{tabular}

We generated normal distribution diagrams of grape TSS values (Figs. 3A and 3D). When the curve is shifted further to the right, this indicates a higher TSS value, and a higher curve apex indicates greater consistency in TSS. The minimum TSS required for high-quality fruit is $18 \%$. Although the TSS of the shaded grapes declined, it reached $18 \%$ under the gray, blue, and green shade nets, indicating that these shade treatments produced high-quality fruits. Moreover, the normal distribution of TSS peaked for the gray shade treatment, indicating that TSS was more consistent under this treatment.

We generated normal distribution diagrams of peel color index (Figs. 3B, E). A normal distribution diagram shifted to the right indicates redder peel color, and a higher curve apex indicates higher color consistency. Hence, peel color was affected by shading, and the black shade net had the greatest 
influence. Redder peel gives "Jumeigui" a better appearance. Gray shade net had the smallest effect on peel color, particularly at $\mathrm{PQ}$.

Fruit firmness showed clear differences across treatments (Fig. 3C, F). At PQ, fruit firmness was highest under blue shade net, followed by gray, black, green, and non-shading treatment. Grape firmness was more consistent under blue shade net than under the other treatments. However, at $\mathrm{ZH}$, fruit firmness was lower under the non-shading treatment than under the shading treatment.

\section{Leaf photosynthetic performance.}

We selected five old leaves near the base and five adult upper leaves near the top, to determine $C i, A, g s$, and $E$ values of the old and adult leaves varied between the treatments. At PQ, the $C i$ of the adult leaves was significantly lower under gray and blue shade net than under the other treatments (Fig. 4A), while the $A$ of the old leaves was significantly lower under the non-shading treatment than under the shading treatment (Fig. 4B). The $g s$ and $E$ of the old leaves were not significantly different between the five treatments (Fig. 4C, D). The $A$ of the adult leaves did not differ significantly among the treatments at PQ (Fig. 4B). The $C i, g s$, and $E$ of the adult leaves were significantly lower under gray and blue shade net than under the other treatments at PQ (Fig. 4A, C, D). In contrast, at ZH, the $A$ of the old leaves was significantly lower, and the $\mathrm{Ci}$ of the old leaves was significantly higher, under the non-shading treatment than under the shading treatments (Fig. 4E, F). The gs and $E$ of the old leaves, and the $C i, A, g s$, and $E$ values of the adult leaves, showed no significant differences across the five treatments (Fig. $4 \mathrm{E}-\mathrm{H}$ ), indicating that the shading treatment had a greater effect on the adult leaves.

At $\mathrm{PQ}$, the $F_{\mathrm{v}} / F_{\mathrm{m}}$ and $P I_{\mathrm{ABS}}$ of the old and adult leaves were significantly lower in the non-shading treatment than under gray and black shade net (Fig. 5A and 5B). In contrast, the $W_{k}$ and $M_{0}$ of the old and adult leaves were significantly higher under the non-shading treatment than under the shading treatments(Fig. 5C and 5D). The $\psi_{\text {Eo }}$ and $\Phi_{\text {EO }}$ of the leaves were significantly lower under the non-shading treatment than under the shading treatment(Fig. $5 \mathrm{E}$ and $5 \mathrm{~F}$ ). The $\delta_{\mathrm{Ro}}$ of the old and adult leaves were highest under the non-shading treatment, being significantly higher than those under black shade net(Fig. $5 G)$.

At $\mathrm{ZH}$, the $F_{\mathrm{v}} / F_{\mathrm{m}}$ and $P I_{\mathrm{ABS}}$ of the adult and old leaves were significantly higher in the shading treatments than in the non-shading treatment, being highest under black shade net(Fig. $5 \mathrm{H}$ and $5 \mathrm{I}$ ). The $W_{\mathrm{k}}$ and $M_{\mathrm{o}}$ were significantly higher in the non-shading treatment than in the other treatments, especially under black and gray shade net(Fig. $5 \mathrm{~J}$ and $5 \mathrm{~K}$ ). In contrast, the $\psi_{\text {Eo }}$ and $\Phi_{\text {Eo }}$ were significantly lower in the nonshading treatment than under black, gray, and green shade net (Fig. $5 \mathrm{~L}$ and $5 \mathrm{M}$ ). Finally, the $\delta_{\mathrm{Ro}}$ value of the old leaves was significantly lower under black shade net than in the other treatments, while the $\delta_{\text {Ro }}$ value of the adult leaves showed no significant differences across the five treatments(Fig. $5 \mathrm{~N}$ ).

\section{Discussion}


According to model predictions, the grape growing season will become warmer, while some cool climate regions are expected to transition to warm or hot by $2080^{17}$. Climate warming has also led to the rapid ripening and leaf aging ${ }^{18}$ but shading treatment can alleviate this problem. Colored shade nets can change the composition of the spectral transmission and reflection ${ }^{6}$. They are widely used and have been shown to improve the microclimate ${ }^{19}$. They can provide an effective strategy in greenhouse cultivation and production.

Chlorophyll is critical in the photosynthetic process. Different wavelengths of light interact with corresponding photoreceptors to regulate the synthesis of different pigments ${ }^{20}$. The absorption wavelength of visible light is mainly concentrated in $430-450 \mathrm{~nm}$ and $640-660 \mathrm{~nm}^{21}$. Therefore we focused on analyzing the light transmittance of the strongest chlorophyll absorption area. Gray and blue shade net has higher light transmittance, producing healthier leaves (Figure S1).

Wu et al. ${ }^{22}$ found that shading can prevent heat-stress damage of grape plants in summer. However, in our study, we examined perennial fruit vines and used a phased shading treatment to achieve ideal cooling and light transmission. We found that the $A$ value of the old leaves was more optimal under the shading treatment than under the non-shading treatment, indicating that long-term heat and strong light stress can cause clear premature aging of the old grape leaves. Further, the trends in the chlorophyll fluorescence parameters and photosynthetic indicators differed, reflecting the immediate response to adversity. Therefore, the $W_{\mathrm{k}}$ and $P I_{\mathrm{ABS}}$ values of the old and adult leaves were more optimal under the shading treatment than under the non-shading treatment. Furthermore, $W_{\mathrm{k}}$, a main indicator for heat stress, is higher at high temperatures, because heat damages the oxygen-evolving complex ${ }^{23}$. In contrast, $P I_{\text {ABS }}$ decreases after heat treatment, reflecting the inhibition of PSII activity ${ }^{2}$. In this study, we found that the non-shading treatment produced the highest $W_{\mathrm{k}}$ value and a low $P I_{\mathrm{ABS}}$ value, indicating that the nonshading treatment caused heat stress. And shading had a positive effect on leaf photosynthesis.

\section{Shading can reduce fruit damage due to heat-induced softening, and ensure fruit quality}

Light and temperature can affect sugar accumulation in wine and table grapes ${ }^{24}$. In our study, the soluble-solid content decreased slightly during the shading treatment, but on average remained $>18 \%$; this satisfies the requirements for high-quality fruit, indicating that the shading treatment reduces the sugar content without affecting its quality.

Anthocyanin is influenced both by environmental factors and viticulture practices. Several studies of grapes have reported the effects of light exposure or shading on anthocyanin accumulation ${ }^{25,26}$. Temperature is another important factor that affects anthocyanin biosynthesis in plants; the expression of anthocyanin biosynthesis genes is inhibited by heat stress ${ }^{27,28}$. This indicates that temperature and light are major factors in determining grape-peel color. Hence, applying the appropriate temperature and light is crucial. Therefore, in this study, we aimed to determine the conditions that reduce the temperature 
without causing excessive shading and provide the most suitable environment for grape growth and development. For this, we found that gray and blue shade nets were ideal for peel coloring.

The effect of shade nets on fruits depends mainly on the cultivar, the environment, and the light utilization efficiency at the canopy level ${ }^{29}$. Gray shades scatter more light, resulting in a more even light distribution on the plants ${ }^{30}$, thereby improving the light use efficiency, photosynthetic efficiency, and dry matter accumulation ${ }^{31}$. For instance, tomato yields are under gray-color shade nets ${ }^{19}$, which can also prevent crops from being exposed to direct sunlight and over-ripening ${ }^{32}$. Using shade net, therefore, delays both harvesting and ripening and can be reflected in the grape quality. In this study, sugar content was lower and peel color was less intense under the shading treatments than under the non-shading treatment, reflecting delayed maturity, consistent with previous reports ${ }^{33,34}$.

\section{Abbreviations}

PSII: photosystem II; TSS: total soluble solids; CIRG: color index for red grapes; OJIP: transient chlorophyll a fluorescence induction.

\section{Declarations}

\section{Acknowledgements}

This work was financed by the National Key Research and Development Program (grant number 2019YFD1001904-09). ZH and PQ are belonged with Shanghai Academy of Agricultural Sciences. And Shanghai Academy of Agriculture Sciences, Shanghai, China was provided permission to collect the plant material.

\section{Author contribution statement}

QZ: Conceptualization; Data curation; Formal analysis; Validation; Methodology; Visualization; Writing original draft. XX: Data curation; Validation. YH: Methodology. XY: Formal analysis; AJ: Writing - original draft.

\section{Conflict of interest}

The authors declare that the research was conducted in the absence of any commercial or financial relationships that could be construed as a potential conflict of interest.

\section{Additional information}


Supplementary information related to this paper can be found, in the online version, at doi:

\section{References}

1. Hale, C. R. \& Buttrose, M. S. Effect of temperature on ontogeny of berries of Vitis vinifera L. Cv. Cabernet Sauvignon. J. Am. Soc. Hort. Sci, 99 (5), 390-394 (1974).

2. Zha, Q., Xi, X., Jiang, A. \& Tian, Y. H. High temperature affects photosynthetic and molecular processes in field-cultivated Vitis vinifera L. $\times$ Vitis labrusca L. Photochem. Photobiol, 92 (3), 446454 (2016).

3. Reynolds, A. G., Pool, R. M. \& Mattick, L. R. Influence of cluster exposure on fruit composition and wine quality of Seyval blanc grapes. Vitis, 25, 85-95 (1986).

4. Xi, X., Zha, Q., He, Y. \& Jiang, A. Influence of cluster thinning and girdling on aroma composition in 'Jumeigui' table grape. Sci. Rep, 10 (1), 1-10 (2020).

5. Puértolas, J., Benito, L. F. \& Peñuelas, J. L. Effects of nursery shading on seedling quality and postplanting performance in two Mediterranean species with contrasting shade tolerance. New. For, $\mathbf{3 8}$ (3), 295 (2009).

6. Shahak, Y. et al. A new approach for light manipulation in fruit trees. Acta. Hort, 636, 609-616 (2004).

7. Martinez-Luscher, J., Chen, C. C. L., Brillante, L. \& Kurtural, S. K. Partial solar radiation exclusion with color shade nets reduces the degradation of organic acids and flavonoids of grape berry (Vitis vinifera L.). J. Agri. Food Chem, 65 (49), 10693-10702 (2017).

8. Warren, J. W., Hand, D. W. \& Hannah, A. Light interception and photosynthetic efficiency in some glasshouse crops. J. Exp. Bot, 43 (3), 363-373 (1992).

9. Smart, R. E., Smith, S. M. \& Winchester, R. V. Light quality and quantity effects on fruit ripening for cabernet sauvignon. Am. J. Enol. Vitic, 39, 250-258 (1988).

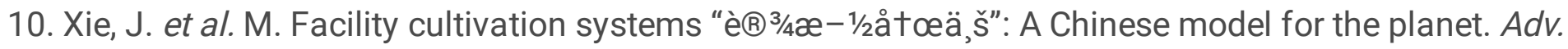
Agron, 145, 2-42 (2017).

11. Zha, Q., Xi, X., He, Y. \& Jiang, A. Bagging Affecting Sugar and Anthocyanin Metabolism in the Ripening Period of Grape Berries. Not. Bot. Hort. Agrob, 47 (4), 1194-1205 (2019).

12. Zhang, Z. et al. Calcium is involved in exogenous NO-induced enhancement of photosynthesis in cucumber (Cucumis sativus L.) seedlings under low temperature. Sci. Hort, 261, 108953 (2020).

13. Giusti, M. \& Wrolstad, R. Characterization and measurement of anthocyanins by UV-visible spectroscopy. Wrolstad R, Schwartz S (Eds.), Current Protocols in Food Analytical Chemistry, John Wiley \& Sons Inc, New York pp. F1.2.1-13F(2001).

14. Lee, J., Durst, R. W. \& Wrolstad, R. E. Determination of total monomeric anthocyanin pigment content of fruit juices, beverages, natural colorants, and wines by the $\mathrm{pH}$ differential method: collaborative study. J. AOAC Inter, 88 (5), 1269-1278 (2005). 
15. Lijavetzky, D. et al. Berry flesh and skin ripening features in Vitis vinifera as assessed by transcriptional profiling. Plos One, 7 (6), e39547 (2012).

16. Amiri, M. E., Fallahi, E. \& Parseh, S. Application of ethephon and ABA at $40 \%$ veraison advance maturity and quality of 'Beidaneh Ghermez' grape. Inter. Sym. Plant Bioreg. Fruit Prod, 884, 371-377 (2010).

17. Hewer, M. J. \& Brunette, M. Climate change impact assessment on grape and wine for Ontario, Canada's appellations of origin. Reg. Environ. Cha, 20 (3), 1-15 (2020).

18. Schultz, H. Climate change and viticulture: A European perspective on climatology, carbon dioxide and UV-B effects. Austr. J. Grape Wine Res, 6 (1), 2-12 (2000).

19. Caroline, L. J., Saidi, M. \& Opiyo, A. Effect of colored agro-net covers on insect pest control and yield of tomato (Solanum lycopersicon Mill). J. Agri. Sci, 9 (12), 59028311 (2017).

20. Fang, S. et al. A novel efficient single-phase dual-emission phosphor with high resemblance to the photosynthetic spectrum of chlorophyll A and B. J. Mate. Chem, 8 (18), 6245-6253 (2020).

21. Terashima, I., Fujita, T., Inoue, T., Chow, W. S. \& Oguchi, R. Green light drives leaf photosynthesis more efficiently than red light in strong white light: revisiting the enigmatic question of why leaves are green. Plant Cell Physiol, 50 (4), 684-697 (2009).

22. Wu, Y. et al. Effects of shading on leaf physiology and morphology in the 'Yinhong' grape plants. Rev. Brasil. Fruticul, 40 (5), e-037 (2018).

23. Zha, Q., Xi, X., He, Y. \& Jiang, A. Water limitation mitigates high-temperature stress injuries in grapevine cultivars through changes in photosystem II efficiency and antioxidant enzyme pathways. Acta. Physiol. Plantar, 41 (6), 83 (2019).

24. Greer, D. H. \& Weston, C. Heat stress affects flowering, berry growth, sugar accumulation and photosynthesis of Vitis vinifera cv. Semillon grapevines grown in a controlled environment. Fun. Plant Biol, 37 (3), 206-214 (2010).

25. Matus, J. T. et al. Post-veraison sunlight exposure induces MYB-mediated transcriptional regulation of anthocyanin and flavonol synthesis in berry skins of Vitis vinifera. J. Exp. Bot, 60 (3), 853-867 (2009).

26. Li, J. et al. Identification and thermal stability of purple-fleshed sweet potato anthocyanins in aqueous solutions with various pH values and fruit juices. Food Chem, 136 (3-4), 1429-1434 (2013).

27. Mori, K., Sugaya, S. \& Gemma, H. Decreased anthocyanin biosynthesis in grape berries grown under elevated night temperature condition. Sci. Hort, 105 (3), 319-330 (2005).

28. Yamane, T., Jeong, S. T., Goto-Yamamoto, N., Koshita, Y. \& Kobayashi, S. Effects of temperature on anthocyanin biosynthesis in grape berry skins. Am. J. Enol. Viticul, 57 (1), 54-59 (2006).

29. Bastías, R. M., Manfrini, L. \& Grappadelli, L. C. Exploring the potential use of photo-selective nets for fruit growth regulation in apple. Chilean. J. Agri. Res, 72 (2), 224 (2012). 
30. Nissim-Levi, A. et al. Light scattering shade net increases branching and flowering in ornamental pot plants. Hort. Sci. Biotech, 83, 9-14 (2008).

31. Shahak, Y., Gal, E., Offi, Y. \& Ben-Yakir, D. Photoselective shade netting integrated with greenhouse technologies for improved performance of vegetable and ornamental crops. International Workshop on Greenhouse Environmental Control and Crop Production in Semi-Arid Regions 797, 75-80(2008).

32. Stamps, R. H. Use of colored shade netting in horticulture. HortScience, 44 (2), 239-241 (2009).

33. Ghiglieno, I. et al. The effects of leaf removal and artificial shading on the composition of Chardonnay and Pinot noir grapes. OENO One, 54 (4), 761-777 (2020).

34. Martin, D. et al. Grape cluster microclimate influences the aroma composition of Sauvignon blanc wine. Food Chem, 210, 640-647 (2016).

\section{Figures}



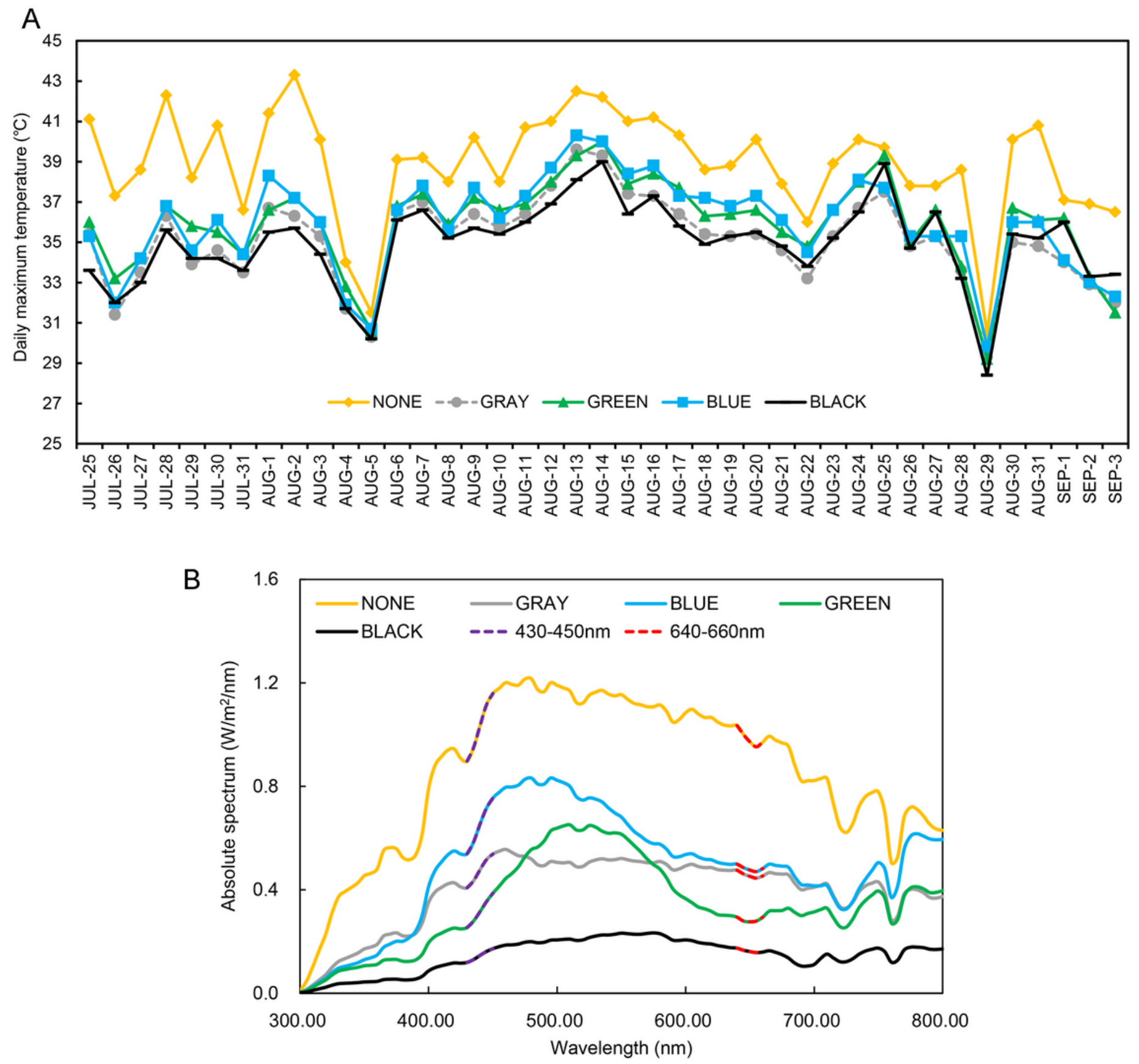

\section{Figure 1}

Processing temperature and spectrum data in the facility greenhouse under the different shading treatments. (A) The daily maximum temperature trend in the facility greenhouse under the colored shading materials. (B) The projected light spectrum composition of the colored shading materials. NONE: no shading; GRAY, BLUE, GREEN, and BLACK: shade net colors 

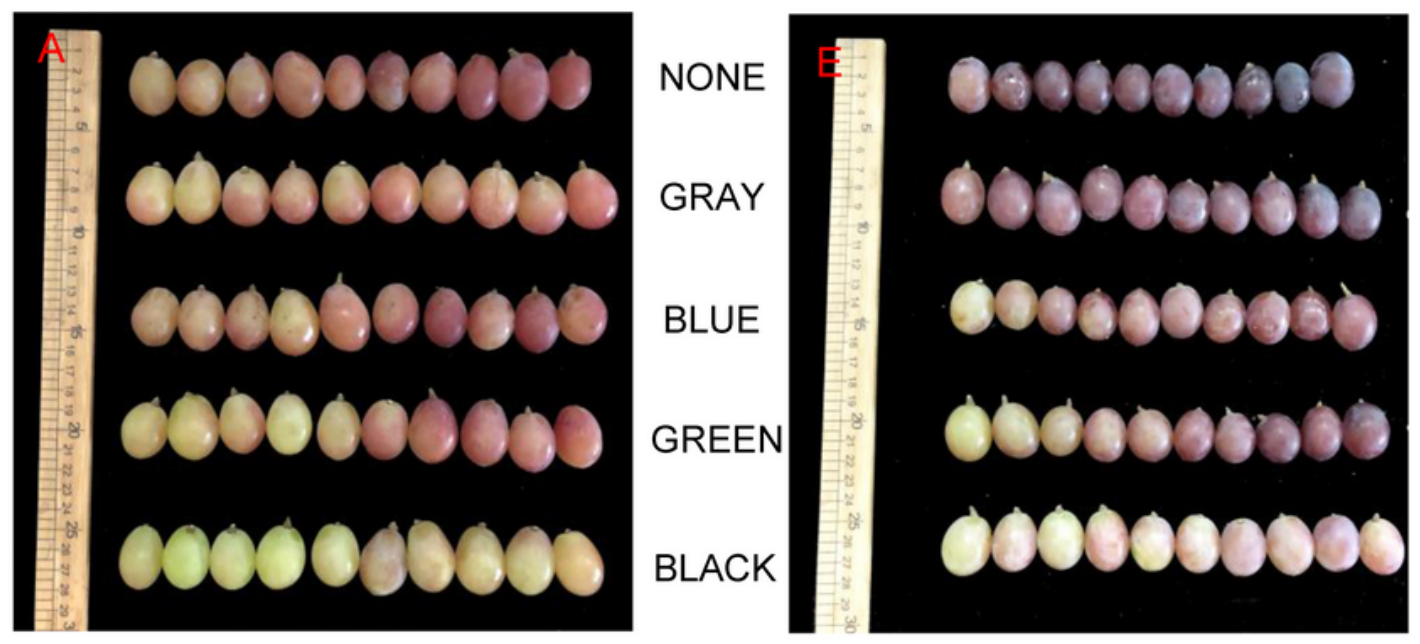

$\mathrm{B}$

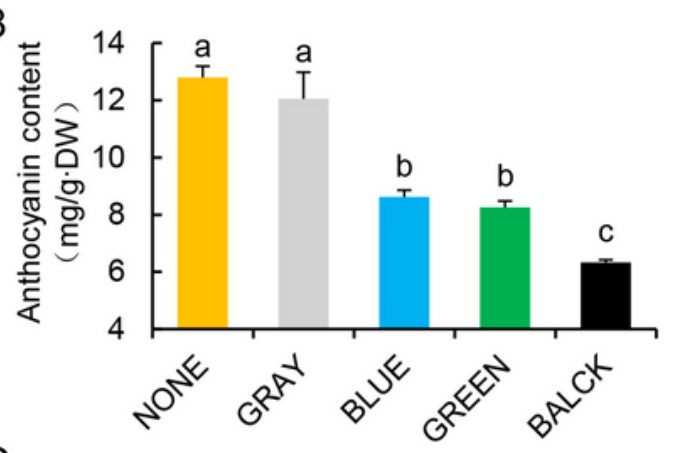

C

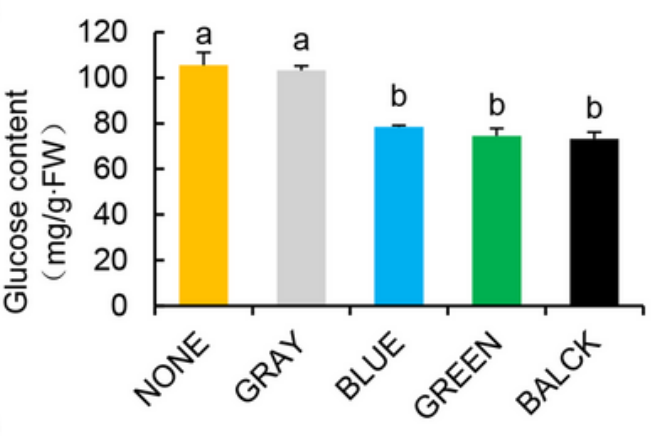

$\mathrm{F}$

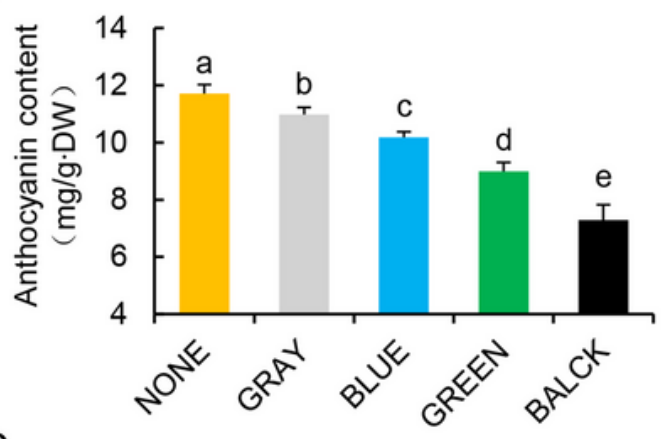

G
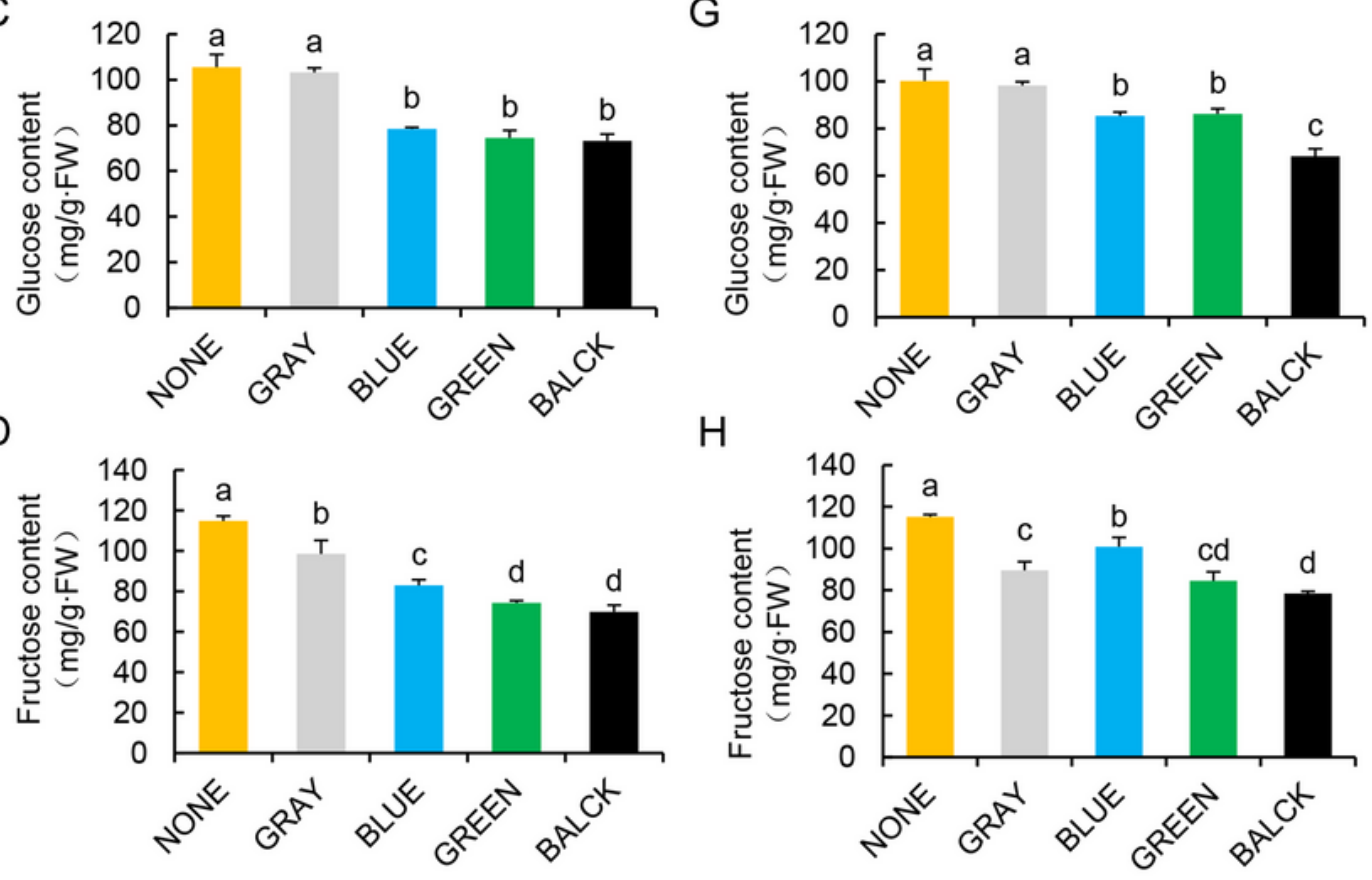

$\mathrm{H}$

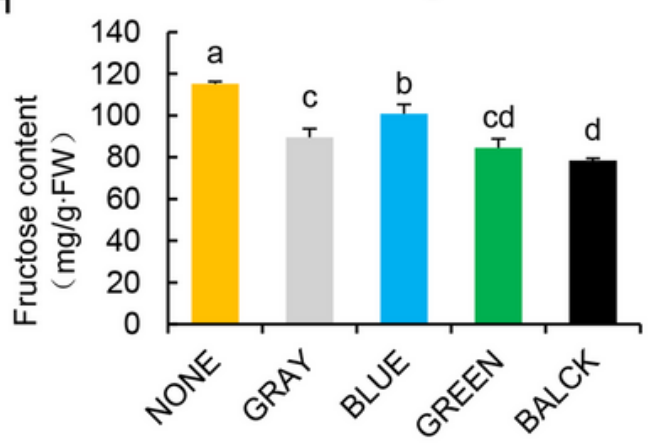

Figure 2

The grapes and their composition across the different treatments. A and E: grape images; Anthocyanin, glucose, and fructose levels at PQ (B-D) and ZH (F-H). NONE: no shading; GRAY, BLUE, GREEN, and BLACK: shade net colors. 

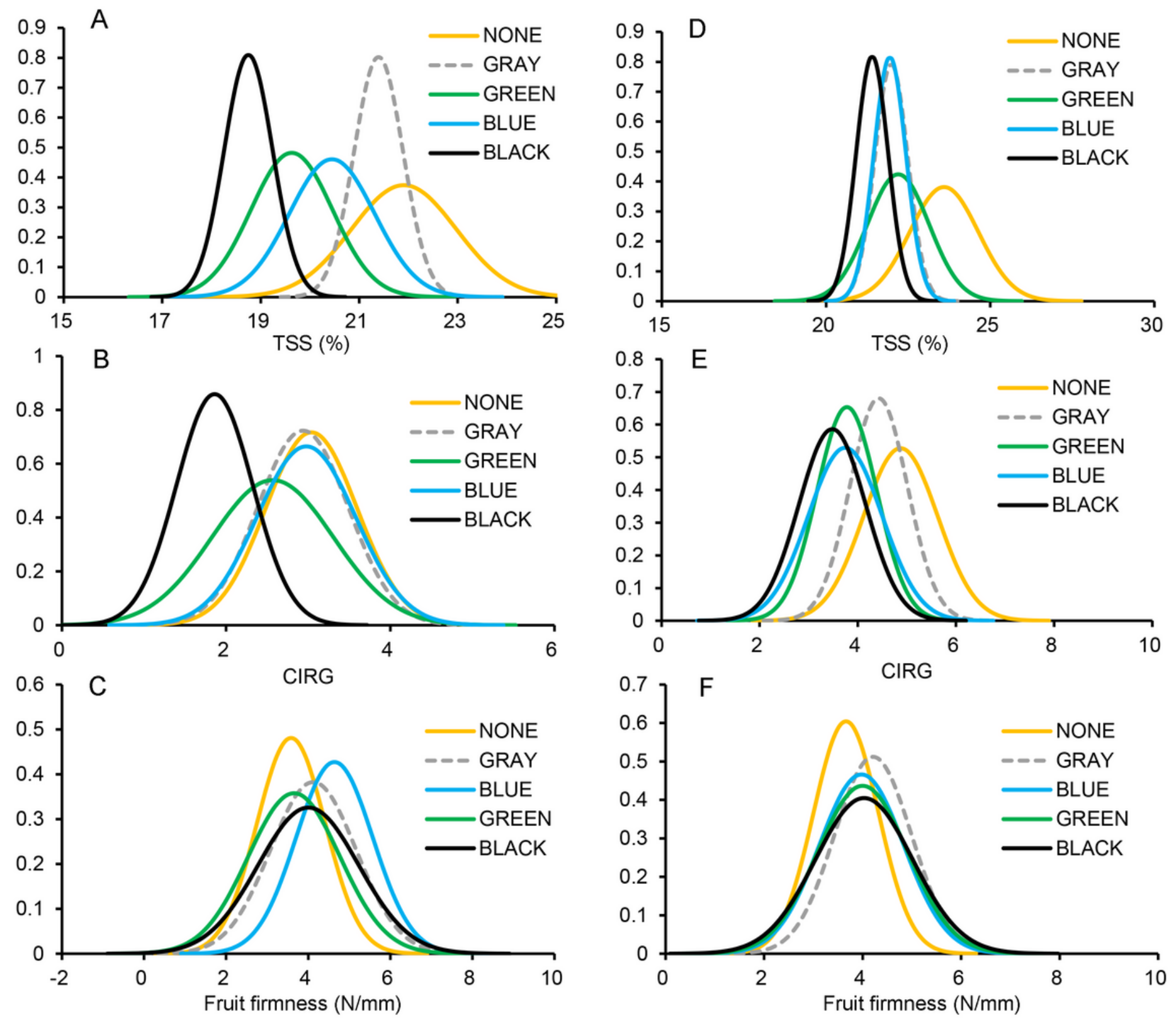

Figure 3

Differences in TSS, CIRG, and fruit firmness across the different treatments. TSS, CIRG, and fruit firmness, at PQ $(A-C)$ and $Z H(D-F)$. NONE: no shading; GRAY, BLUE, GREEN, and BLACK: shade net colors. 


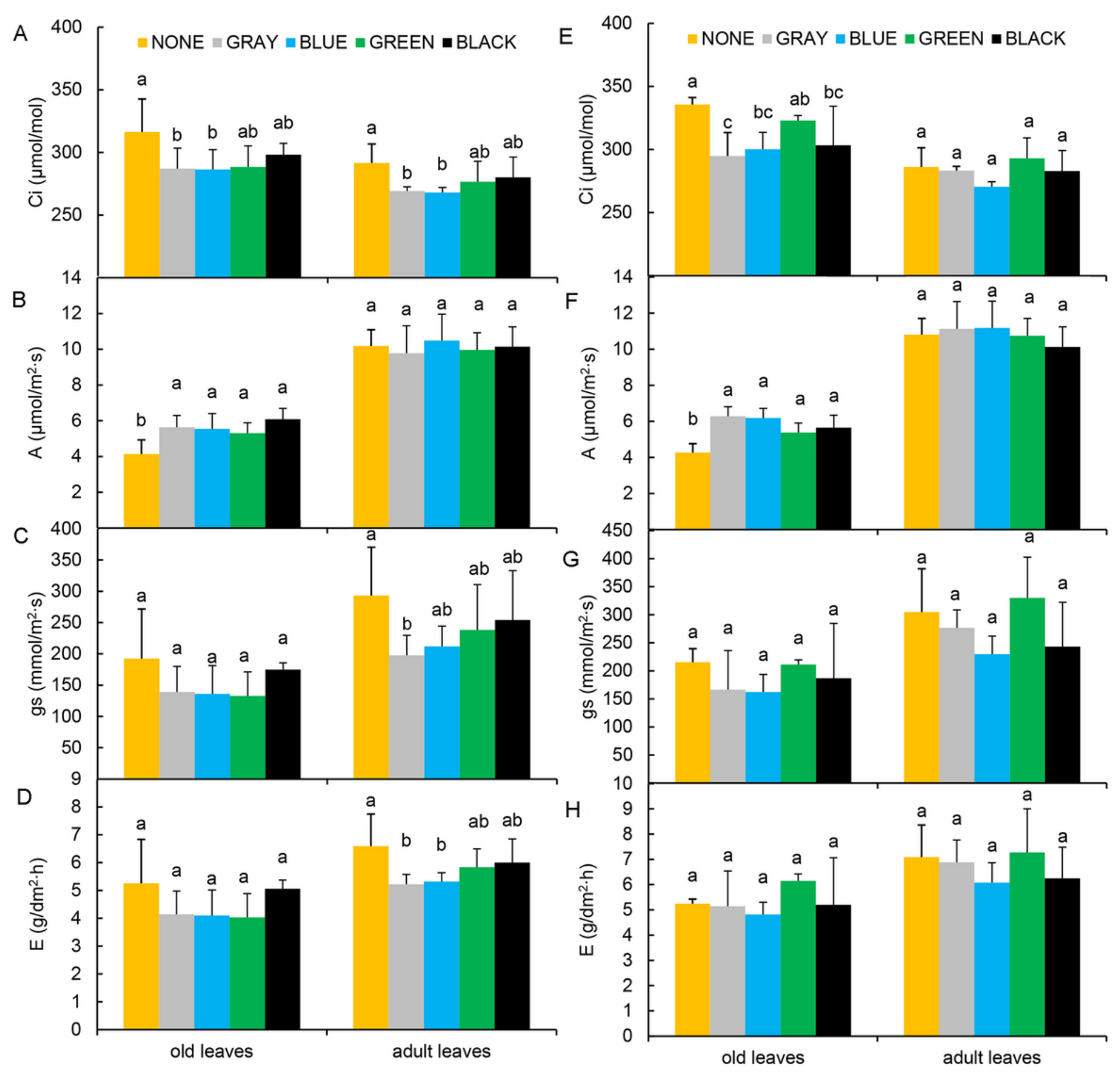

Figure 4

Differences in the photosynthesis-related parameters of the grapevine leaves across the different treatments. $\mathrm{Ci}, \mathrm{A}$, gs, and $E$ across the different treatments at $P Q(A-D)$ and $\mathrm{ZH}(E-H)$. NONE: no shading; GRAY, BLUE, GREEN, and BLACK: shade net colors. 

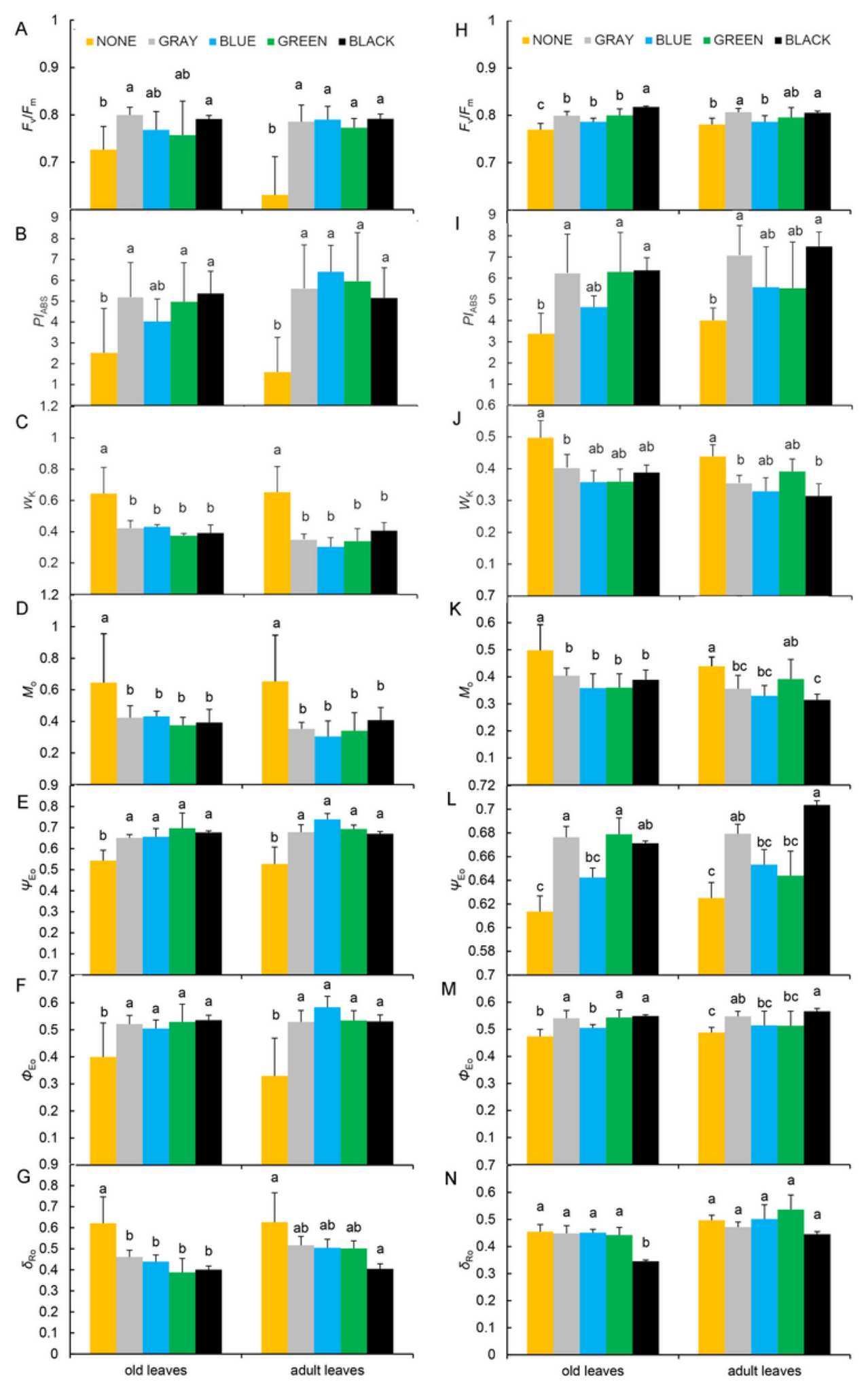

Figure 5

Differences in PSII-related parameters of grapevine leaves under the different treatments. Fv/Fm, PIABS, Wk, Mo, $\Psi E O, \Phi E o$, and $\delta$ Ro at PQ $(A-G)$ and $Z H(H-N)$. NONE: no shading; GRAY, BLUE, GREEN, and BLACK: shade net colors.

\section{Supplementary Files}


This is a list of supplementary files associated with this preprint. Click to download.

- SupplementaryMaterial.docx 